Cereal Research Communications 43(2), pp. 318-325 (2015)

DOI: $10.1556 / C R C .2014 .0047$

First published online 4 February, 2015

\title{
Molecular Detection of the 1BL.1RS Translocation in Hellenic Bread Wheat Cultivars
}

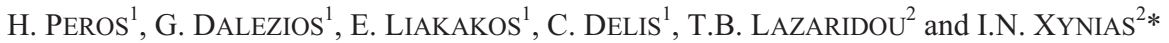 \\ ${ }^{1}$ T. E. I. of Peloponnese, Lab of Genetics and Plant Breeding, School of Agricultural Technology \& \\ Food Technology and Nutrition, Antikalamos, 24100 Kalamata, Hellas \\ ${ }^{2}$ T. E. I. of Western Macedonia, Lab of Genetics, School of Agricultural Technology \& Food Technology \\ and Nutrition, 53100 Florina, Hellas \\ (Received 8 July 2014; Accepted 18 August 2014; \\ Communicated by P.S. Baenziger)
}

\begin{abstract}
The main motive of the present work was to detect and confirm the presence of the 1BL.1RS wheat-rye translocation in elite Hellenic bread wheat germplasm. For this, a molecular method was employed and seven Hellenic bread wheat cultivars (Acheloos, Acheron, Chios, Elisavet, Myconos, Orpheus, and Vergina) were studied. A rye population originated from Florina, and cultivar Kavkaz/Cgn, one of the donors of the 1BL.1RS translocation to bread wheat, were used as positive checks. The polymerase chain reaction was applied and two promoters for bread wheat gene alleles located on short arm of chromosome 1B (GLU-B3, $G L I-B 3)$ and one promoter for a rye gene (SEC I) located on short arm of chromosome 1R, were involved. It was demonstrated that cultivars Acheron, Elisavet and Orpheus do carry the 1BL.1RS translocation, whereas the rest of the varieties studied, they do not it. It was concluded that cultivars Acheron, Elisavet and Orpheus that carry the translocation, could be crossed to other elite genotypes lucking the translocation and produce new varieties possessing the advantages of the 1BL.1RS translocation. A special attention should be given to cultivar Acheron since this cultivar, despite the presence of the translocation carries favourable alleles in other gene loci affecting positively the quality of the produced bread.
\end{abstract}

Keywords: 1BL.1RS translocation, polymerase chain reaction

\section{Introduction}

The presence of the 1BL.1RS wheat rye chromosome translocation in many modern bread wheat cultivars attracted the interest of breeders broadly (Tang et al. 2009). This is because the translocated fragments of rye chromosome offer to the host cultivar a number of important agronomic traits, i.e. high yield potential (Kim et al. 2004), due to increased seed production and seed weight especially under water efficient conditions (Ehdaie et al. 2003), broad adaptation (Rajaram et al. 1983; Schlegel and Meinel 1994), resistance to drought, due to a broader root system developed under drought conditions and an in-

\footnotetext{
*Corresponding author; E-mails: ioannis_xynias@hotmail.com; xynias@teikal.gr
} 
creased proportion root/shoot dry weight (Hoffmann 2008), and biotic stress conditions (Bartos and Bares 1971; Zeller 1973; Moreno-Sevilla et al. 1995) and increased regeneration capacity of the plants (Rabinovich 1998), contributing to a sustainable wheat production. The 1BL.RS translocation is originated from cv. Kavkaz, and according to Weng et al. (2007) possesses resistance genes to stem rust (Puccinia graminis Pers), stripe rust (Puccinia striiformis Westend), leaf rust (Puccinia recondita Rob. Ex Desm) and powdery mildew (Erisiphe graminis DC). More precisely, the short arm of 1R chromosome contains resistance genes against leaf rust ( $\operatorname{Lr} 26)$, stem rust $(\mathrm{Sr} 31)$, stripe rust $(\mathrm{Yr} 9)$ and powdery mildew (Pm8) (Schneider and Molnar-Lang 2008). The main disadvantages of the translocation are that the aforementioned genes are linked with decreased bread-making-quality (Graybosch et al. 1993; Fenn et al. 1994). Their action, however, depends on the genetic background of the host cultivar (Rabinovich 1998; Dimitrijevic et al. 2008) and the prevailing environmental conditions. Finally and most important, they are no longer effective against new biotypes of the diseases (Schneider and Molnar-Lang 2008). However, other results demonstrate a considerable resistance of some new Hellenic doubled haploid lines carrying the translocation (lines obtained through anther-culture) not only to rusts and mildew but to Septoriasis as well under the conditions of Ukraine (Lisova et al. 2005).

It is obvious that the important agronomic advantages of the translocation are the ones that motivated its wide use. As it is referred by Hoffmann (2008) in Hungary 53\% of the cultivated wheat varieties during the past 20 years, carry the short arm of the first rye chromosome. From 1991 to $1995,45 \%$ of 505 commercial cultivars from 17 countries (Rabinovich 1998) and up to 50\% of all wheat cultivars in China (Zhou et al. 2003) carry the 1BL.1RS wheat-rye translocation. In CIMMYT 70\% of spring wheat germplasm carry the translocation; however, this proportion was declined to about $30 \%$ in more recent advanced lines (Singh et al. 2006). In addition, recent reports state a loss of effectiveness of the resistant genes to biotic stress conditions (Schneider and Molnar-Lang 2008; Tang et al. 2008). This is attributed to the wide use of the 1BL.1RS translocation, and it is suggested that new sources of resistant genes must be obtained (Tang et al. 2008). The presence of the 1BL.1RS wheat rye translocation can be detected with biochemical and molecular markers (Xynias et al. 2007; Varzakas et al. 2014). Seed storage proteins are the most common biochemical marker in wheat and an extended review on their application in wheat breeding was published by Xynias et al. (2007). Molecular markers on the other side are useful tools that have been used to identifying the presence of the short arm of the $1 \mathrm{R}$ rye chromosome (Wang et al. 2009). This suggests that certain markers, related to the $1 \mathrm{RS}$ arm can be used in marker assisted selection (MAS) facilitating the introduction of the 1 RS in commercial wheat cultivars. Kim et al. (2005) referred to the existing interest for the introgression of the 1RS into durum wheat germplasm. Fu et al. (2010) identified using the PCR method, 100 wheat lines, resulting after monosomic addendum of the 1 RS arm, originating from the Chinese rye cultivar R12. Regarding the improved resistance of the obtained lines 1R734, 1R314 and 1R725, the chromosome arm 1R of the aforementioned rye cultivar could represent a novel and valuable gene source in improving the resistance of bread wheat to diseases. According to Lukaszewski (2000) central transloca- 
tions of the 1RS arm are useful in bread wheat breeding because they insure resistance to different parasites and diseases and increased grain yield. Their main disadvantage is the reduced bread-making quality.

The aim of the present study was to detect the presence of the 1BL. 1RS translocation in modern Hellenic commercial varieties of bread wheat with the polymerase chain reaction approach. This detection could facilitate interpreting the performance of this germplasm to various stress conditions.

\section{Materials and Methods}

\section{Plant material}

Seven spring bread wheat cultivars (cvs. Acheloos, Acheron, Orpheus, Elisavet, Vergina, Myconos, and Chios), broadly cultivated in Hellenic Republic, were used in this study. Five of the aforementioned cultivars were bred in NAGREF-Cereal Institute of Thessaloniki (cvs. Acheloos, Acheron, Orpheus, Elisavet and Vergina), and two in the Laboratory of Genetics and Plant Breeding of the Aristotle University of Thessaloniki (cvs. Myconos and Chios). The above cultivars are important not only for their agronomic value, but also because they are widely used in current breeding programs: cv. Vergina is the best adapted to the Hellenic climatic conditions, cultivars Acheloos, Acheron and Elisavet are newer varieties, with excellent bread making quality, whereas cv. Orpheus is a cold resistant variety grown in certain areas of the country. In addition cvs Acheron, and Acheloos are very well responding to anther culture and for this they are broadly used in breeding programs. These cultivars were also used because three of them (cvs "Acheron", Orpheus and Elizavet) when examined with biochemical markers, they were found to carry the 1BL.1RS translocation while a forth one (cv. Chios) was found to be a mixture of two genotypes, one of which carried the former translocation (Xynias et al. 2006). Finally, cv. Kavkaz/Cgn (one of the donators of the 1BL.1RS wheat rye translocation) which was kindly provided by the Hellenic Gene Bank and a spring rye population originating from Florina were used as checks.

\section{Sample preparation}

Seeds of all bread wheat (Triticum aestivum L. em Thell) varieties and the rye (Secale cereale L.) population used were sterilized for $5 \mathrm{~min}$ in ethanol, rinsed with sterile water and then were transferred for $15 \mathrm{~min}$ in $5 \% \mathrm{NaOCl}-0.02 \%$ Tween 20 solution and finally rinsed at least ten times with sterile distilled water. The seeds were pregerminated for eight days between several sterile sheets of moist paper, in Petri dishes, for two days in the dark and then transferred in 12/12 light/dark photoperiod at $22{ }^{\circ} \mathrm{C}$. For all samples genomic DNA was isolated with Nucleospin plant (Macherey-Nagel, Duren, Germany) according to manufacturer's instructions. For bread wheat varieties Orpheus, Acheron, KVZ/Cgn and Elisavet three biological repeats containing roots of ten germinated seedlings, were polled together and ground in liquid nitrogen. For varieties of Chios, Acheloos and 
Vergina 20 individual plants were used for DNA extraction. For positive control of PCR reactions genomic DNA was extracted from rye plants.

\section{Detection of $1 B L / 1$ RS chromosomal translocation}

1BL/1RS chromosomal translocation was detected in bread wheat varieties by PCR method. Specific primers were designed against GLIB1 (GLI-B1F: 5'-CTTGCCCT CCTTGCTATCG-3' and GLIB1R: 5'-TGTTGTTCTTGTTGTTGTTGTTT-3') and SEC1 (SEC1F:5'-GAGTCATCAGTCGTTATCG-3' and SEC1R: 5'-AAATGGTTGT TGTGTTTGC-3') genes located in1BS and 1RS chromosomes of bread wheat and rye respectively (Fig. 1). PCR was performed by Kapa2G FAST PCR kit according to manufacturer's instructions. PCR amplicons were monitored on agarose gel electrophoresis on a $1.5 \%(\mathrm{w} / \mathrm{v})$ gel. As positive control genomic DNA from the rye population was used.

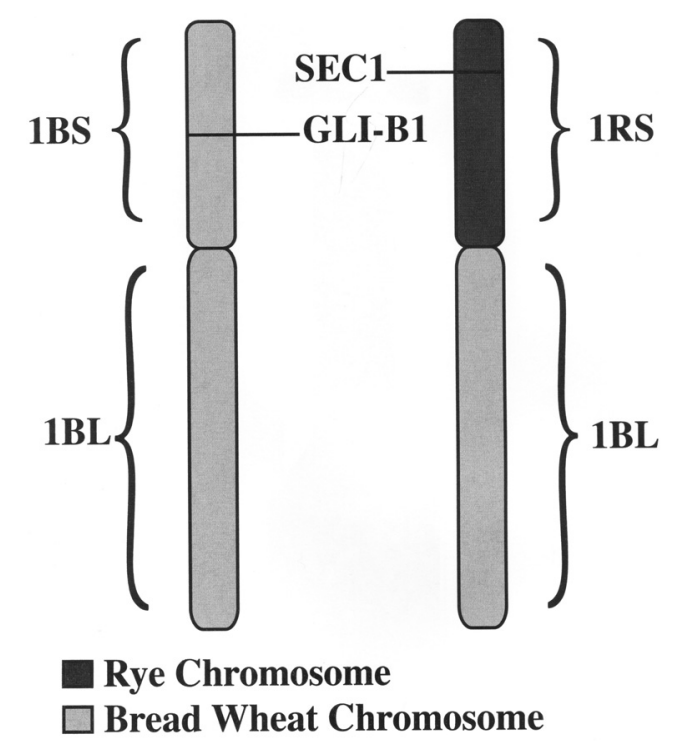

Figure 1. The normal 1B chromosome (left) and the translocated (right) and two corresponding storage protein genes

\section{Results}

The presence of $S E C 1$ gene which is typical of the short arm of $1 \mathrm{R}$ chromosome of rye was detected in four of the examined cultivars (cvs. Orpheus, Acheron, Kavkaz/Cgn and Elisavet, Fig. 2). In two cultivars (cvs. Acheloos and Vergina), no detection of the aforementioned gene was observed (Fig. 3).

Cultivar Chios it was examined in more details, since in a previous research it was found to be consisted of two different genotypes, one carrying the translocation (Xynias et al. 2006). For this, 20 individual plants were subjected to PCR. In all cases the SEC1 gene 


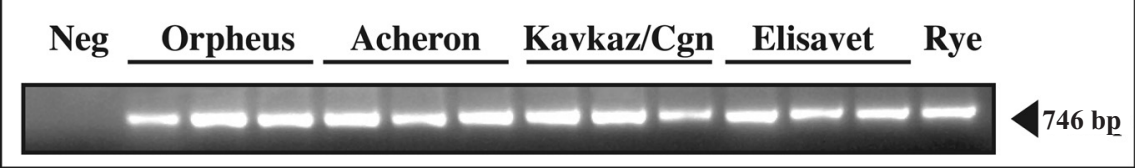

Figure 2. The detection of SECl gene in four bread wheat varieties (Orpheus, Acheron, Kavkaz/Cgn and Elisavet). Neg indicates the negative control for PCR

Acheloos

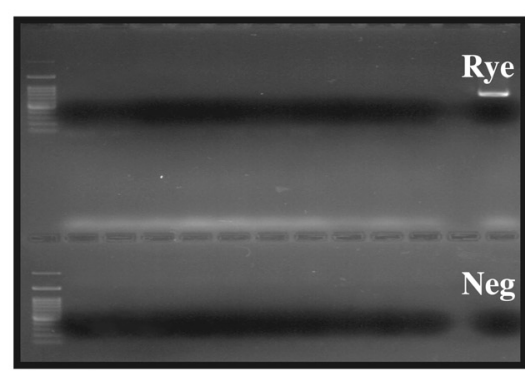

\section{Vergina}

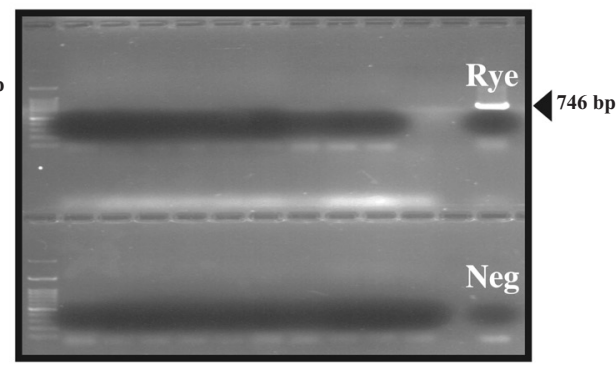

Figure 3. The detection of SEC1 gene on 20 individual plants of Acheloos and Vergina bread wheat varieties. Neg indicates the negative control

was not detected suggesting that cultivar Chios does not possess rye genomic DNA (Fig. 4A). In contrast, the presence of GLI-BIgene, which is a typical gene of the short arm of 1B was detected in all plants studied (Fig. 4B).

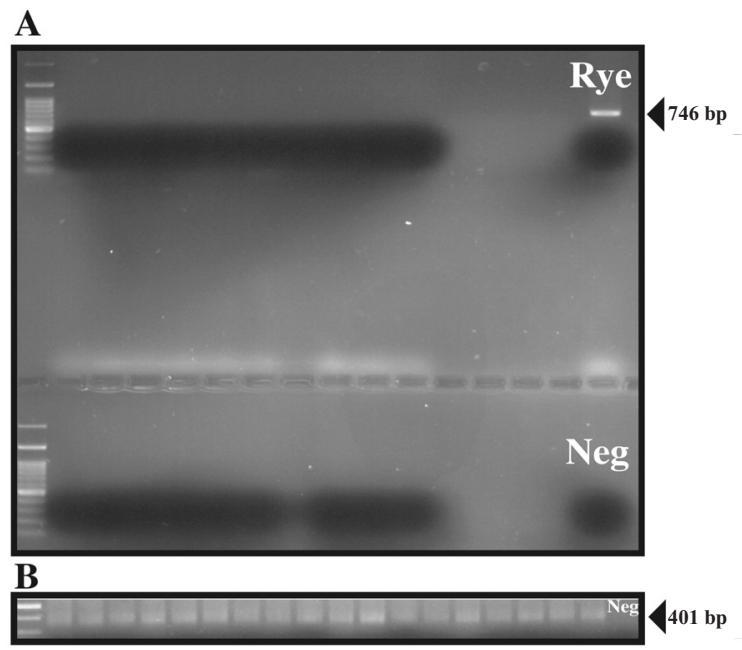

Figure 4. The detection of SECI (A) and GLI-BI (B) genes on 20 individual plants of Chios bread wheat variety. Neg indicates the negative control in both (A) and (B) figures 


\section{Discussion}

The presence of the 1BL.1RS wheat-rye translocation is still one of the main goals in current breeding programs. However, recent data suggest that in order to benefit the advantages of the translocation, the genetic constitution of the host cultivar plays an important role (Dimitrijevic et al. 2008). Lisova et al. (2005) presented data to support this view by screening doubled haploid lines (DHL), progenies of the same cross, carrying the translocation. They reported that two lines (DHL 16 and DHL 17), originating from the cross between the Hellenic cultivar Penios and the Russian cultivar Kavkaz/Cgn proved to be resistant to the local populations in Ukraine of powdery mildew, leaf rust and septoriosis during all the vegetation stages. DHL 18, progeny of the same cross, was susceptible to leaf rust when seedlings were properly inoculated. This may indicate that the genetic constitution of the cultivar might be important in exploiting the advantages of the presence of 1 RS arm.

The present work verifies previous reports that molecular markers facilitate indeed the detection of the translocation (Wang et al. 2009). The aforementioned markers are accurate and reliable, since they detect certain DNA, typical of rye genome. The authors of the present study believe that they are a more reliable tool than the biochemical markers. This is because the biochemical markers detected the presence of the translocation in a proportion of plants in cultivar Chios (Xynias et al. 2006) something that it is not true. However, if the positive advantages of the translocation are to be exploited more effectively then more sources of the translocation must be utilized in different genetic backgrounds (Dimitrijevic et al. 2008).

As long as the bread-making quality is concerned, Lelley et al. (2004) concluded that the value of the 1BL.1RS translocation for wheat breeding at its present form is questionable, because of its negative effects on gluten and bread making quality. According to Graybosch et al. (1993) the 1AL.1RS wheat rye translocation has fewer deleterious effects on baking quality. They further consider 1AL.1RS translocation as a more favorable vehicle than the T1BL.1RS for the utilization of rye genes and enhancing of wheat performance. However, Mckendry et al. (2001) reported a more pronounced negative effect on quality of $1 \mathrm{AL} .1 \mathrm{RS}$ than the respective of 1BL.1RS. This was further supported in a more recent article in which it was referred that if the rye source will be carefully selected, then quality defects can be kept to a minimum level, and 1BL.1RS translocation (along with 1DL.1RS) are better choices for soft wheat quality (Kim et al. 2005). It is unquestionable that wheat bread making quality is affected by more than one gene loci (Xynias et al. 2007). Thus, if the host wheat cultivar possesses a favorable gene constitution then it becomes quite feasible to introduce the translocation with no detrimental effects on quality. Cultivar Acheron is a good example of the last case (Xynias et al. 2006). Another alterative, in order to avoid the negative effects of the translocation, is transferring of small rye chromosome segments into wheat germplasm (Ribeiro-Carvalho et al. 1997). Such minor translocations may occur spontaneously and can be maintained by local farmers as landraces due to their high agronomic value. 
In conclusion, the 1BL.1RS translocation is still of great importance, especially under the prevailing drought conditions in the southern part of Europe. The molecular detection of the presence of the translocation is a very effective and accurate tool, but more research is needed to exploit the positive effects of the translocation in host-wheat germaplasm.

\section{References}

Bartos. R., Bares, I. 1971. Leaf and stem rust resistance of hexaploid wheat cultivars Salzmunder Bartweizen and Weique. Euphytica 20:435-440.

Dimitrijevic, M., Petrovic, S., Gustafson, J.P. 2008. The effect of wheat/rye translocation 1BL/1RS in a different quality genetic background on biological traits in wheat. Genetika 40:261-270.

Ehdaie, B., Whitkus, R.W., Waines, J.G. 2003. Root biomass, water-use efficiency and perfomance of wheat-rye translocations of chromosomes 1 and 2 in spring bread wheat 'Pavon'. Crop Science 43:710-717.

Fenn, D., Lukow, O.M., Bushuk, W., Depauw, R.M. 1994. Milling and baking quality of 1BL/1RS translocation wheats. I. Effects of genotype and environment. Cereal Chem. 71:189-195.

Fu, S., Tang, Z., Ren, Z., Zhang, H., App, J. 2010. Transfer to wheat (Triticum aestivum) of small chromosome segments from rye (Secale cereale) carrying disease resistance genes. Genet. 51:115-121.

Graybosch, R.A., Peterson, C.J., Hansen, L.E., Worrall, D., Shelton, D.R., Lukaszewski, A. 1993. Comparative flour quality and protein characteristics of $1 \mathrm{BL} / 1 \mathrm{RS}$ and $1 \mathrm{AL} / 1 \mathrm{RS}$ wheat-rye transnlocation lines. J. Cereal Sci. 17:95-106.

Hoffmann, B. 2008. Alteration of drought tolerance of winter wheat caused by translocation of rye chromosome segment 1RS. Cereal Res. Commun. 36:269-278.

Kim, W., Johnson, J.W., Baenziger, P.S., Lukaszewski, A.J., Gaines, C.S. 2004. Agronomic effect of wheat-rye translocation carrying rye chromatin (1R) from different sources. Crop Sci. 44:1254-1258.

Kim, W., Johnson, J.W., Baenziger, P.S., Lukaszewski, A.J., Gaines, C.S. 2005. Quality effect of wheat-rye (1R) translocation in 'Pavon 76'. Plant Breeding 124:334-337.

Lelley, T., Eder, C., Grausgruber, H. 2004. Influence of 1BL.1RS wheat-rye chromosome translocation on genotype by environment interaction. J. Cereal Sci. 39:313-320.

Lisova, G.M., Kozub, N.O., Sozinov, I.O., Xynias, I.N. 2005. Donors of leaf rust, powdery mildew, and Septoriosis resistance among Greek bread wheat cultivars and dihaploid lines. Plant Protection and Quarantine 51:22-29. (In Russian with English abstract)

Lukaszewski, A.J. 2000. Manipulation of the 1RS.1BL translocation in wheat by induced homoeologous recombination. Crop Sci. 40:216-225.

Mckendry, A.L., Tague, D.N., Ross, K. 2001. Comparative effects of 1BL.1RS and 1AL.1RS on soft red winter wheat milling and baking quality. Crop Sci. 41:712-720.

Moreno-Sevilla, B., Baenziger, P.S., Shelton, D., Graybosch, R.A., Peterson, C.J. 1995. Agronomic performance and end-use quality of $1 \mathrm{~B}$ and $1 \mathrm{~B} / 1 \mathrm{R}$ genotypes derived from winter wheat Rawhide. Crop Sci. 35:1607-1622.

Rabinovich, S.V. 1998. Importance of wheat-rye translocation for breeding modern cultivars of Triticum aestivum L. Euphytica 100:323-340.

Rajaram, S., Mann, C.E., Ortis Ferrara, G., Mujeeb-Kazi, A. 1983. Adaption, stability and high yield potential of certain 1B/1R CIMMYT wheats. In: Sakamoto, S. (ed.), Proc. $6^{\text {th }}$ Int. Wheat Genet Symp. Plant Germ-Plasm Institute, Kyoto, Japan, pp. 613-621.

Ribeiro-Carvalho, C., Guedes-Pinto, H., Harrison, G., Heslop-Harrison, J.S. 1997. Wheat-rye chromosome translocations involving small terminal and intercalary rye chromosome segments in the Portuguese wheat landrace Barbela. Heredity 78:539-546.

Schlegel, R., Meinel, A. 1994. A quantitative trait locus (QTL) on chromosome arm 1RS of rye and its effects on yield performance of hexaploid wheat. Cereal Res. Commun. 22:7-13.

Schneider, A., Molnar-Lang, M. 2008. Polymorphism analysis using 1RS-specific molecular markers in rye cultivars (Secale cereale L.). Cereal Res. Commun. 36:11-19.

Singh, R.P., Hodson, D.P., Jin, Y., Huerta-Espino, J., Kinyua, M.G., Wanyera, R., Njau, P., Ward, R.W. 2006. Current status, likely migration and strategies to mitigate the threat to wheat production from race Ug99

Cereal Research Communications 43, 2015 
(TTKS) of stem rust pathogen (Review). CAB Reviews: Perspectives in Agriculture, Veterinary Science, Nutrition and Natural Resources 2006 1, No. 054. http://www.ars.usda.gov/SP2UserFiles/Place/ 36400500/PAV054.pdf

Tang, Z.X., Fu, S.L., Ren, Z.L., Zhang, H.Q., Yang, Z.J., Yan, B.J., Zhang, H.Y. 2008. Production of a new wheat cultivar with a different 1B.1R translocation with resistance to powdery mildew and stripe rust. Cereal Res. Commun. 36: 451-460.

Tang, Z.X., Fu, S.L., Ren, Z.L., Zhang, H.Q., Yang, Z.J., Yan, B.J. 2009. Characterization of three wheat cultivars possessing new 1BL.1RS wheat-rye translocations. Plant Breeding 128:524-527.

Varzakas, T., Kozub, N., Xynias, I.N. 2014. Quality determination of wheat: Genetic determination, biochemical markers, seed storage proteins - bread and durum wheat germplasm. Journal of the Science of Food and Agriculture. DOI: $10.1002 /$ jsfa.6601

Wang, C.M., Li, L.H., Zhang, X.T., Gao, Q., Wang, R.F., An, D.G. 2009. Development and application of EST-STS markers specific to chromosome 1RS of Secale cereale. Cereal Res. Commun. 37:13-21.

Weng, Y., Azhaguvel, P., Devkota, R.N., Rudd, J.C. 2007. PCR-based markers for detection of different sources of 1AL.1RS and 1BL.1RS wheat-rye translocations in wheat background. Plant Breeding 126:482-486.

Xynias, I.N., Kozub, N.O., Sozinov, I.A. 2006. Seed storage protein composition of Hellenic bread wheat cultivars. Plant Breeding 125:408-410

Xynias, I.N., Kozub, N., Sozinov, I., Sozinov, A. 2007. Biochemical markers in wheat breeding. Int. J. Plant Breeding 1:1-9.

Zeller, F. 1973. 1B/1R wheat-rye chromosome substitutions and translocations. In: Sears, E.R., Sears, L.M.S (eds), Proc. $4^{\text {th }}$ Int. Wheat Genetics Symposium. Missouri Agricultural Experimental Station. Columbia, OH, USA, pp. 209-222

Zhou, Y., He, Z.H., Liu, J.J., Liu, L. 2003. Distribution of 1BL/1RS translocation in Chinese winter wheat and its effect on noodle quality. In: Pogna, N.E., Romano, M., Pogna, E., Galterio, G. (eds), Proc. $10^{\text {th }}$ Int. Wheat Genetics Symposium. Paestum, Italy, vol. 3, pp. 1419-1421. 